\title{
IPA NEWS
}

TREASURER'S REPORT 2008-2010

Year ending 31st December 2008

INCOME

Subscriptions

$£ 8,868$

CUP

$£ 17,833$

Examination fees

$£ \quad 625$

Interest

$£ 1,343$

Total

$£ 28,669$

Surplus

$£ 27,019$

Balance in current account: $£ 11,767$

Balance in reserve account: $£ 63,172$

Year ending 31st December 2009

\section{INCOME}

Subscriptions

$£ 10,143$

CUP

$£ 4,766$

Examination fees

$£ \quad 250$

Interest

$£ \quad 559$

Total

$£ 15,718$

Surplus

$£ 5,934$

OUTGOINGS

Bank charges $\quad £ \quad 61$

Examiner fees $\quad £ 459$

CUP Journal $\quad £ 4,534$

Web design $\quad £ 318$

Software $\quad £ 103$

Editorial $\quad £ 2,309$

Admin $\quad £ 2,000$

$£ 9,784$

Balance in current account: $£ 13,388$

Balance in reserve account: $£ 67,391$

Year ending 31st December 2010

INCOME

Subscriptions

$£ 6,360$

CUP

$£ 4,479$

Examination fees

$£ 600$

Interest

$£ 575$

Total

$£ 12,014$

Surplus

$£ 3,623$

OUTGOINGS

Bank Charges

$£ \quad 69$

Examiner fees

$£ 308$

CUP Journal

$£ 5,512$

Editorial

$£ 2,502$

$£ 8,391$

Balance in current account: $£ 7,366$

Balance in reserve account: $£ 71,716$

Dollar account:

$\$ 406$

Euro account:

$€ 8,100$ 
GÖSTA BRUCE MEMORIAL FUND (included in the bank accounts above), ending 31st December 2010: $€ 3,429$

$$
\begin{array}{ll}
£ & 97 \\
\$ & 96
\end{array}
$$

Additional contributions after December 2010 brought the total to $€ 6,436$ and $\$ 200$.

Twelve Gösta Bruce Studentships of $€ 300$ were awarded this year, for travel to the 2011 $\mathrm{ICPhS}$.

A more detailed breakdown is available on request.

\section{Linda Shockey}

Outgoing Treasurer 